\title{
Chronic liver disease and subchronic nephritis in a male warty chameleon (Furcifer verrucosus) with transient hyperglycaemia - case report
}

\author{
Zdeněk Knotek ${ }^{1,2}$, Zora Knotková1, Šárka Trnková1, Gerry M. Dorrestein ${ }^{1,3}$, \\ William Lewis ${ }^{4}$ \\ ${ }^{1}$ Avian and Exotic Animal Clinic, Faculty of Veterinary Medicine, \\ University of Veterinary and Pharmaceutical Sciences, \\ Brno, Czech Republic \\ ${ }^{2}$ Clinic for Avian, Reptile and Fish Medicine, University of Veterinary Medicine, Vienna, Austria \\ ${ }^{3}$ Diagnostic Pathology Laboratory NOIVBD, Veldhoven, The Netherlands \\ ${ }^{4}$ The Wylie Veterinary Centre, Upminster, Essex, United Kingdom
}

Received November 1, 2010

Accepted November 15, 2011

\begin{abstract}
A two-year old male warty chameleon (Furcifer verrucosus) weighing $160 \mathrm{~g}$ was presented for veterinary examination following 4 weeks of decreased ability to catch insects with the tongue and difficulty in swallowing the prey. Non-invasive endoscopy did not reveal any macroscopic changes of the oral cavity mucosa or the cranial part of the esophagus. Dorsoventral and laterolateral plain and contrast radiographs revealed enlargement of the medial part of the liver without any visible abnormalities in the regions of the esophagus, stomach or small intestine. Abnormalities in the plasma chemistry profile included transient hyperglycaemia $(52.68-57.18$ $\mathrm{mmol} / \mathrm{l})$ and hyperuricaemia (452.70-622.20 $\mu \mathrm{mol} / \mathrm{l})$. The chameleon was examined at 7, 20 and 22 weeks after initial examination. Its body weight decreased to $120 \mathrm{~g}$. A blood profile revealed normoglycaemia $(16.37-10.22 \mathrm{mmol} / \mathrm{l})$ and hyperphosphataemia $(2.92-3.06 \mathrm{mmol} / \mathrm{l})$ at the last three examinations. The chameleon died suddenly 33 days after the final examination. Necropsy revealed the presence of a large liver cyst, filled with fluid. The liver had lost all of its normal structure. The kidneys showed a large area with fibrosis and multiple uric acid tophi. The post mortem findings were defined as liver with fatty degeneration and moderate fibrotic changes with large cyst, subchronic nephritis with uric acid tophi, and mineralization in the myocardium. This paper describes the first documented case of transient hyperglycaemia in a warty chameleon (Furcifer verrucosus) associated with chronic liver disease and subchronic nephritis.
\end{abstract}

Reptiles, kidneys, hepatic failure, glucose, plasma chemistry

A limited number of studies have described nutritional and metabolic diseases of chameleons (Kik 1995; Knotek et al. 2008). A tentative diagnosis of chronic liver disease could be considered in reptiles (including chameleons) if blood profile analyses reveal a transient hyperglycaemia and the possible influence of stress could be ruled out (Frye 1991). This study describes the first documented case of transient hyperglycaemia in a warty chameleon (Furcifer verrucosus) associated with chronic liver disease and subchronic nephritis.

\section{Materials and Methods}

A two-year old male warty chameleon (Furcifer verrucosus) weighing $160 \mathrm{~g}$ was presented for veterinary examination following 4 weeks of decreased ability to catch insects with the tongue and difficulties in swallowing the prey. Nutrition and husbandry practices in the owner's collection of different species of chameleons and agamid lizards were considered above average to excellent based on the history. The diet consisted of different species of invertebrates mixed with mineral dust and vitamin powder. The owner reported restricted motility of the chameleon's tongue.

Physical examination revealed a good body condition, but a decreased level of activity of the chameleon. Abdominal palpation showed a residual content in the gastrointestinal tract, gentle external palpation of the body near the pelvic region excluded renomegaly. After the clinical examination, the animal was hospitalized for

Address for correspondence:

Prof. MVDr. Zdeněk Knotek, CSc.

Avian and Exotic Animal Clinic, Faculty of Veterinary Medicine,

University of Veterinary and Pharmaceutical Sciences, Brno,

Czech Republic
Phone: +420604205305

Fax: +42541562381

E-mail: knotekz@vfu.cz

http://www.vfu.cz/acta-vet/actavet.htm 
3 days. Blood samples $(0.6 \mathrm{ml})$ were collected at arrival and at 4 days after hospitalization and after 7, 20 and 22 weeks after the first visit to the clinic. The packed cell volume (PCV) was measured by the microhaematocrit, haemoglobin $(\mathrm{Hb})$ was determined by the cyanmethaemoglobin method using a spectrophotometer (Unicam Helios Epsilon, Thermospectronic USA), and the total red and white blood cell counts were done according to the Natt and Herrick method (Knotek et al. 2002; Pejřilová et al. 2004). Blood smears were air-dried and stained using May-Grünwald and Giemsa-Romanowski stains. Two hundred leukocytes were counted for each smear and classified as heterophils, eosinophils, basophils, lymphocytes, azurophils and monocytes (Pejřilová et al. 2004). The blood sample in the tube containing $0.01 \mathrm{ml}$ heparin (5000 i.u./ml Heparin Leciva inj., Prague, Czech Republic) was centrifuged and plasma biochemical assays were performed within $2 \mathrm{~h}$ after collection by the use of automated analyzers (Knotková et al. 2005). The concentration of total protein (TP), glucose, uric acid, phosphorus (P), and the activity of alkaline phosphatase (ALP), alanine aminotransferase (ALT), aspartate aminotransferase (AST), and creatinphosphokinase (CPK), were determined with a CobasMira Plus Analyzer (Roche, France); the plasma concentration of calcium (Ca) was determined by the spectrophotometer (AA Series Spectrometer, Thermo electron corporation, UK). Radiographic examination of the chameleon included a dorsoventral projection (DV) and a laterolateral (LL) view in sternal position by horizontal beam using Proteus XR (GE Medical Systems, USA) and digital automatic system FCR Capsula XL (Fuji, Japan). A gastrointestinal contrast radiographic study was conducted using $1.6 \mathrm{ml} \mathrm{25 \%}$ barium sulphate (Micropaque susp., Delpharm, France) orally as the contrast medium.

\section{Endoscopy}

Induction of anaesthesia was achieved using of $0.03 \mathrm{ml}$ tiletamine-zolazepam $(10 \mathrm{mg} / \mathrm{kg}$ IM, Zoletil 50, Virbac, France) and the chameleon was then intubated. Under isoflurane anaesthesia a detailed physical examination of the tongue, oral cavity and cranial part of the esophagus was performed using a rigid endoscope (Hopkins Documentation Forward-Oblique Telescope 64017 B, ø 1.9-2.1 mm, 19 cm; Karl Storz Tuttlingen, Germany).

\section{Post mortem examination and histopathology}

The post mortem examination was performed within $6 \mathrm{~h}$ after the animal died at the clinic. The tongue, heart, liver, and kidneys were fixed in buffered formalin (10\%). The fixed tissues were routinely processed and after embedding in paraffin $4 \mu \mathrm{m}$ thin slides were cut and stained with haematoxylin-eosin (HE) and microscopically evaluated at the NOIVBD in Veldhoven, the Netherlands.

\section{Results}

Dorsoventral (DV) and laterolateral (LL) plain and contrast radiographs excluded any visible abnormalities in the regions of the esophagus, stomach and small intestine but an enlargement of the medial part of the liver was apparent (Plate V, VI, Figs 1, 2). Endoscopy did not reveal any macroscopic changes in the oral cavity, tongue or the cranial part of the esophageal mucosa. The results of the blood profile of the chameleon are presented in Table 1. A complete blood count revealed azurophilia and lymphopaenia at the first visit (day 1 and 4). Abnormalities of the plasma chemistry profile at the first visit included marked hyperglycaemia and increased concentrations of uric acid.

After 3 days of hospitalization the chameleon was discharged and the owner was advised to keep the lizard in optimal conditions until the next examination. The chameleon underwent follow up examinations 7, 20 and 22 weeks after initial presentation. During the physical examinations the chameleon exhibited active movement and improved overall activity in comparison with the first visit. The use of the tongue was very much improved. The body weight, however, decreased to $120 \mathrm{~g}$ at the last visit. A blood profile revealed normoglycaemia $(16.37,10.61$ and $10.22 \mathrm{mmol} / 1$, respectively) and hyperphosphataemia $(2.92,3.06$ and $3.01 \mathrm{mmol} / 1$, respectively). Thirty three days after the last clinical examination the chameleon died.

\section{Post mortem and histological findings}

At post mortem examination the chameleon was in a poor condition (body weight $105 \mathrm{~g})$. At microscopic examination the very complex organized muscle system of the tongue showed only very few muscle-fibres with old regenerating muscle damage (Plate VI, Fig. 3). The myocardium showed an irregular pattern of vacuolization, mini-fragmentations and swelling of nuclei. There was a 'layer' of mineralization foci at the boundary of the more solid outer wall of the ventricle and the more trabecular inner part. Surrounding 
Table 1. Blood profile of the warty chameleon (Furcifer verrucosus)

\begin{tabular}{lccccc}
\hline Value & \multicolumn{5}{c}{ Blood samples } \\
& Day 1 & Day 4 & +7 weeks & +20 weeks & +22 weeks \\
\hline Haemoglobin $(\mathrm{g} / \mathrm{l})$ & 88 & 85 & 63 & - & - \\
PCV $(1 / 1)$ & 0.30 & 0.22 & 0.34 & 0.30 & 0.28 \\
RBC $\left(10^{12} / 1\right)$ & 1.220 & 0.820 & 1.110 & 1.250 & 1.200 \\
WBC $\left(10^{9} / 1\right)$ & 12.00 & 9.50 & 7.50 & 6.50 & 5.00 \\
Heterophils $\left(10^{9} / 1\right)$ & 5.88 & 4.75 & 3.00 & 2.02 & 2.05 \\
Eosinophils $\left(10^{9} / 1\right)$ & 0 & 0 & 0 & 0 & 0 \\
Basophils $\left(10^{9} / 1\right)$ & 0 & 0 & 0 & 0.07 & 0.05 \\
Monocytes $\left(10^{9} / 1\right)$ & 0.84 & 0.57 & 0.53 & 0.07 & 0.10 \\
Azurophils $\left(10^{9} / 1\right)$ & 4.68 & 3.14 & 1.50 & 0.46 & 0.25 \\
Lymphocytes $\left(10^{9} / 1\right)$ & 0.60 & 1.04 & 2.48 & 3.90 & 2.55 \\
Total protein $(\mathrm{g} / \mathrm{l})$ & 51.40 & 47.20 & 64.20 & 48.00 & 47.80 \\
Glucose $(\mathrm{mmol} / 1)$ & 52.68 & 57.18 & 16.37 & 10.61 & 10.22 \\
ALP $(\mu \mathrm{kat} / \mathrm{l})$ & 5.55 & 5.57 & 10.36 & 2.84 & 2.58 \\
ALT $(\mu \mathrm{kat} / \mathrm{l})$ & $<0.01$ & $<0.01$ & $<0.01$ & 0.01 & 0.02 \\
AST $(\mu \mathrm{kat} / \mathrm{l})$ & 0.24 & 0.05 & 0.12 & 0.05 & 0.03 \\
CPK $(\mu \mathrm{kat} / \mathrm{l})$ & 2.60 & 7.01 & 12.14 & 16.85 & 16.46 \\
Uric acid $(\mu \mathrm{mol} / \mathrm{l})$ & 452.70 & 622.20 & 343.00 & 244.20 & 257.50 \\
Calcium $(\mathrm{mmol} / \mathrm{l})$ & 2.92 & 3.56 & 2.68 & 2.27 & 2.19 \\
Phosphorus $(\mathrm{mmol} / \mathrm{l})$ & 2.88 & 2.31 & 2.92 & 3.06 & 3.01 \\
\hline
\end{tabular}

ALP - alkaline phosphatase, ALT - alanine aminotransferase, AST - aspartate aminotransferase, CPK - creatine phosphokinase, PCV - packed cell volume, RBC - red blood cells, WBC - white blood cells

membranes were thickened, partially hyperaemic and some contained mineralized foci (Plate VI, Fig. 4). The most salient finding was a large liver cyst (diameter $2.5 \mathrm{~cm}$ ) filled with clear fluid. The liver had completely lost its normal structure: the acinar structure was largely lost, hepatocytes showed vacuolization, necrosis and multinuclear regeneration patterns. There was moderate fibrosis and the capsule was slightly thickened. In the liver several small bacterial abscesses with a central bacterial colony of rod-shaped bacteria surrounded by a layer of multinuclear cells within a thin capsule were present (Plate VII, Fig. 5). The kidneys showed a large area with fibrosis and multiple uric acid tophi. Some of these tophi also showed mineralization (Plate VII, Fig. 6).

The post mortem findings were summarized as a liver with (fatty) degeneration and moderate fibrotic changes with large cyst(s), a (sub)chronic nephritis with uric acid tophi, and mineralization in the myocardium.

\section{Discussion}

Many physiological as well as pathological factors can affect blood glucose concentrations in reptiles. The main clinical signs noted by the owners of reptiles suffering from hyperglycaemia are anorexia, lethargy and weakness (Frye 1991). Of particular clinical importance in the reptile patient is stress-associated hyperglycaemia (Stahl 2006). However, the male warty chameleon was kept in very good conditions, with assisted feeding done by its owner. The reported value for glucose concentration $(23.76 \mathrm{mmol} / \mathrm{l})$ in a stressed lizard (Mayer et al. 2005) was significantly lower than those in the first period of this study. Stress related hyperglycaemia, therefore, was not the likely cause of the transient hyperglycaemia in this patient. 
Persistent hyperglycaemia in lizards (Iguana iguana and Physignathus cocincinus) has been seen in cases of chronic renal disease, hepatic lipidosis and diabetes mellitus (Heatley et al. 2001; Crocker and Miller 2002). A five-year old male green iguana with persistently elevated blood glucose suffered from anorexia and abnormalities of its blood chemistry profile including increased AST, phosphorus and creatinphosphokinase. The results of the biochemistry panel in the chameleon were different in many respects to the above mentioned case. In both cases hyperuricaemia was found but the extreme values measured in the green iguana were not seen in our patient. The chameleon also did not suffer from pronounced hyperphosphataemia as was the case in the green iguana with end-stage renal failure. The spontaneous resolution of the hyperglycaemia in the peripheral blood at 7, 20 and 22 weeks without therapeutic intervention makes the diagnosis of diabetes mellitus in the warty chameleon highly unlikely.

Based on the histopathological findings, the warty chameleon was diagnosed a liver with fatty degeneration, moderate fibrotic changes with large cysts, and subchronic nephritis. Based on the cases found in the literature and discussed here, the transient hyperglycaemia in this chameleon is supposed to be caused by these pathological changes. The problems in using the tongue at the first visit may have been the result of muscular damage of which an indication was seen as regenerating scars in the histopathology.

\section{Acknowledgment}

This project received partial support from the University of Veterinary and Pharmaceutical Sciences Brno (Specific research/2008/FVL).

\section{References}

Crocker C, Miller D 2002: Persistent elevated blood glucose in the iguana, Iguana iguana: A case study. Proc ARAV, Reno, 7-9

Heatley JJ, Johnson A, Tully T, Mitchell M 2001: Persistent hyperglycemia in a chinese water dragon, Physignathus cocincinus. Proc ARAV, Orlando, pp. 207-211

Knotek Z, Dorrestein GM, Knotková Z, Jekl V, Grabensteiner E, Trnková Š 2008: Haematology and plasma chemistry in female veiled chameleons (Chamaeleo calyptratus) suffering from pre-ovulatory follicle stasis (POFS). Proc. EAZWV, Leipzig, pp. 189-195

Knotek Z, Hauptman K, Knotková Z, Hájková P, Tichý F 2002: Haemogram and plasma biochemistry in green iguanas with renal disease. Acta Vet Brno, 71: 333-340

Kik MJL 1995: Nutritional, infectious and environmental diseases of chameleons. Proc. Coll Pathol Rept Amphibians, Alphen aan den Rijn, pp. 191-196

Knotková Z, Dorrestein GM, Jekl V, Janoušková J, Knotek Z 2008: Fasting and post-prandial serum bile acid concentrations in 10 healthy female red-eared terrapins (Trachemys scripta elegans). Vet Rec 163: 510-514

Knotková Z, Pejřrilová S, Trnková S, Matoušková O, Knotek Z 2005: Influence of reproductive season upon plasma biochemistry values in green iguanas. Acta Vet Brno 74: 515-520

Mayer J, Knoll J, Innis C, Mitchell MA 2005: Characterizing the hematologic and plasma chemistry profiles of captive Chinese water dragons, Physignathus cocincinus. J Herp Med Surg 15: 16-23

Pejřilová S, Knotková Z, Knotek Z, Vrbas J 2004: Age-related changes of the hematological profile in green iguana (Iguana iguana rhinolopha). Acta Vet Brno 73: 305-312

Stahl SJ 2006: Hypeglycemia in reptiles. In: Mader DR (Ed.), Reptile Medicine and Surgery. $2^{\text {nd }}$ ed., Saunders Elsevier, St. Louis, pp. 822-835 
Plate V

Knotek Z. et al.: Chronic liver ... pp. 397-400

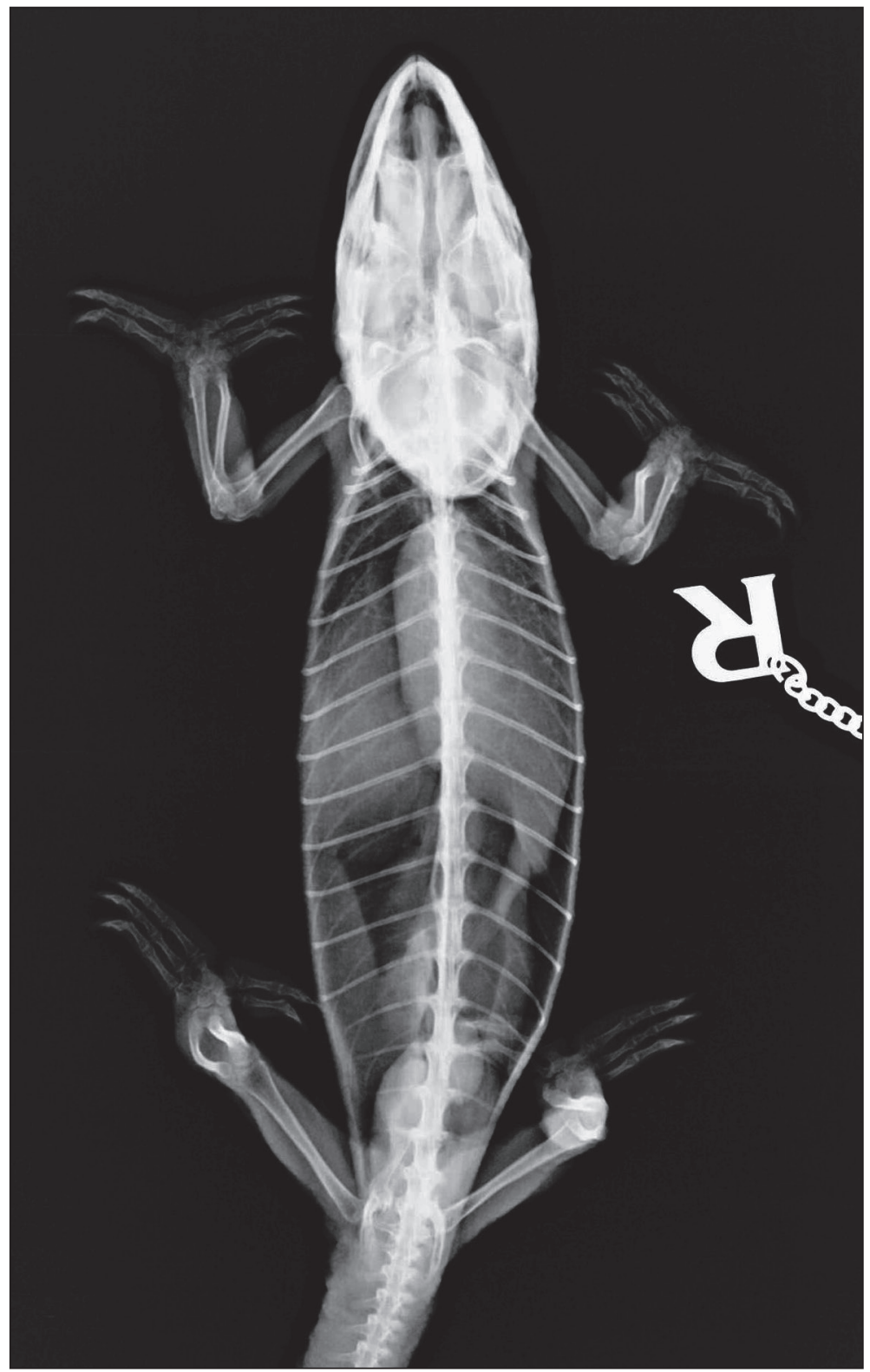

Fig. 1. Dorsoventral radiograph of the chameleon. 


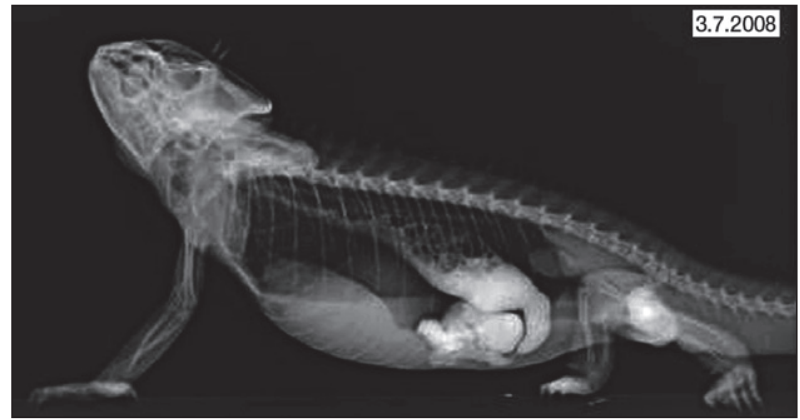

Fig. 2. Laterolateral contrast radiograph with barium sulphate.

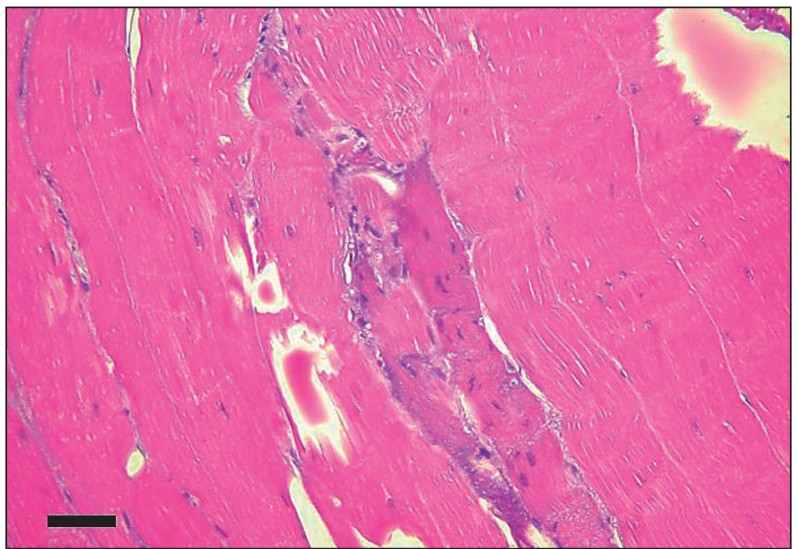

Fig. 3. Histopathology of the chameleon tongue showing scar of muscle fibres. $\mathrm{HE} \times 10$. Bar $50 \mu \mathrm{m}$.

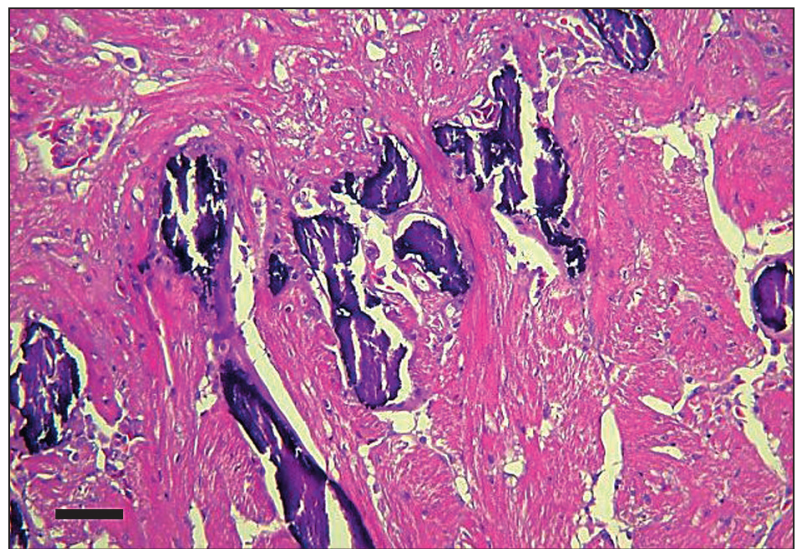

Fig. 4. The chameleon myocardium with a 'layer' of mineralization foci. $\mathrm{HE} \times 10$. Bar $50 \mu \mathrm{m}$. 


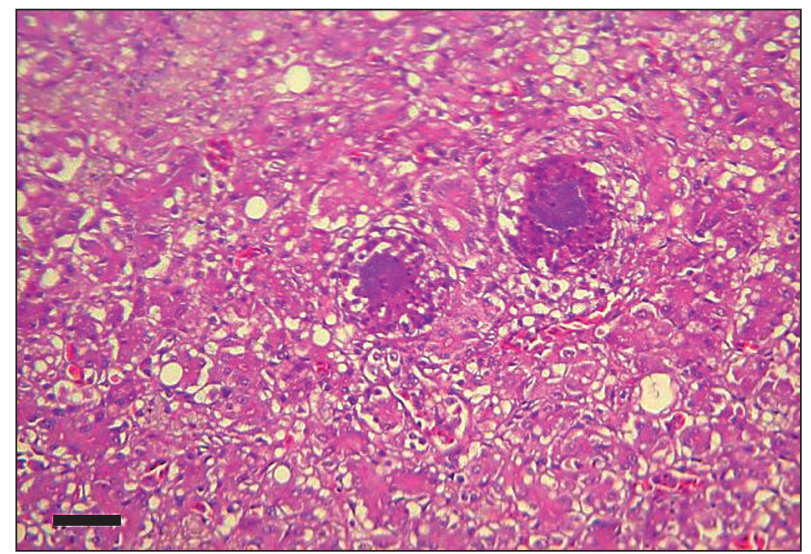

Fig. 5. Several small bacterial abscesses in the chameleon liver. $\mathrm{HE} \times 10$. Bar $50 \mu \mathrm{m}$.

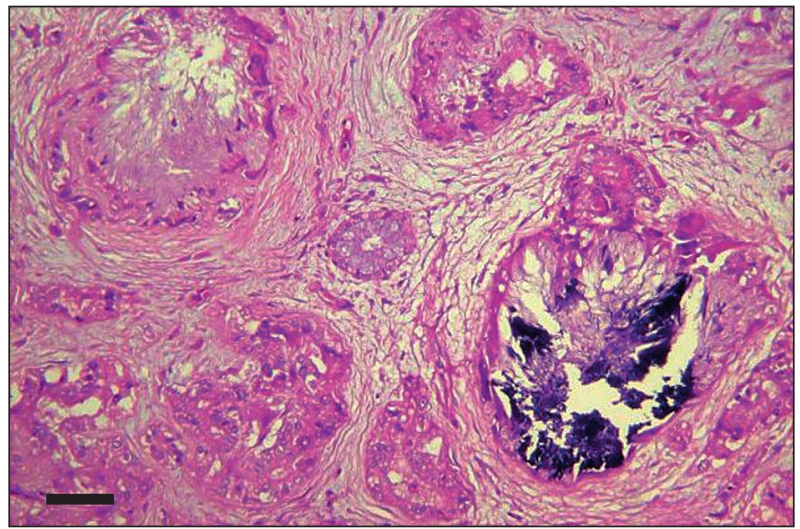

Fig. 6. The chameleon kidneys. Uric acid tophi with mineralization. $\mathrm{HE} \times 10$. Bar $50 \mu \mathrm{m}$ 